Modeling and Simulation Challenges Pursued by the Consortium for Advanced Simulation of Light Water Reactors (CASL)

Paul J. Turinsky ${ }^{\mathrm{a}, *}$

Douglas B. Kothe ${ }^{\text {b }}$

${ }^{a}$ CASL Chief Scientist, North Carolina State University, PO Box 7926, Raleigh, NC 27695-7926 turinsky@ncsu.edu

${ }^{\mathrm{b}}$ CASL Director Emeritus, Oak Ridge National Laboratory, PO Box 2008, Oak Ridge, TN 37831-6164 kothe@ornl.gov

*Corresponding author 
Abstract: The Consortium for the Advanced Simulation of Light Water Reactors (CASL), the first Energy Innovation Hub of the Department of Energy, was established in 2010 with the goal of providing modeling and simulation (M\&S) capabilities that support and accelerate the improvement of nuclear energy's economic competitiveness and the reduction of spent nuclear fuel volume per unit energy, and all while assuring nuclear safety. To accomplish this requires advances in M\&S capabilities in radiation transport, thermal-hydraulics, fuel performance and corrosion chemistry. To focus CASL's R\&D, industry challenge problems have been defined, which equate with long standing issues of the nuclear power industry that M\&S can assist in addressing. To date CASL has developed a multi-physics "core simulator" based upon pin-resolved radiation transport and subchannel (within fuel assembly) thermal-hydraulics, capitalizing on the capabilities of high performance computing. CASL's fuel performance M\&S capability can also be optionally integrated into the core simulator, yielding a coupled multi-physics capability with untapped predictive potential. Material models have been developed to enhance predictive capabilities of fuel clad creep and growth, along with deeper understanding of clad oxidation and hydrogen pickup. Understanding of corrosion chemistry (e.g., CRUD formation) has evolved at all scales: micro, meso and macro. CFD R\&D has focused on improvement in closure models for subcooled boiling and bubbly flow, and the formulation of robust numerical solution algorithms. For multiphysics integration, several iterative acceleration methods have been assessed, illuminating areas where further research is needed. Finally, uncertainty quantification and data assimilation techniques, based upon sampling approaches, have been made more feasible for practicing nuclear engineers via R\&D on dimensional reduction and biased sampling. Industry adoption of CASL's evolving M\&S capabilities, which is in progress, will assist in addressing long-standing and future operational and safety challenges of the nuclear industry.

Keywords: Modeling, simulation, core simulator, multiphysics, nuclear energy 


\title{
Modeling and Simulation Challenges Pursued by the Consortium for Advanced Simulation of Light Water Reactors (CASL)
}

\author{
Paul J. Turinsky - CASL Chief Scientist \\ North Carolina State University, PO Box 7926, Raleigh, NC 27695-7926 turinsky@ncsu.edu \\ Douglas B. Kothe - CASL Director Emeritus \\ Oak Ridge National Laboratory, PO Box 2008, Oak Ridge, TN 37831-6164 kothe@ornl.gov
}

1. Overview

In 2010, the first Energy Innovation Hub of the Department of Energy (DOE) was established: the Consortium for the Advanced Simulation of Light Water Reactors (CASL) ${ }^{1}$. Energy Innovation Hubs bring together teams of top scientists and engineers from academia, industry, and government to collaborate and overcome critical known barriers to achieving national climate and energy goals that have proven resistant to solution via the normal R\&D enterprise. Hubs focus on a single topic, with the objective of rapidly bridging the gaps between basic research, engineering development, and commercialization through a close partnership with industry [1]. To achieve this goal, the Hubs necessarily consist of large, highly integrated and collaborative creative teams working to solve priority technology challenges. Hubs basically strive to enable and accelerate translational research-a high return proposition for nuclear energy.

For CASL the focus is on enhancing innovation for commercial nuclear power generation, specifically for modeling and simulation (M\&S) of nuclear reactors. CASL not only strives to bring innovation to the nuclear energy enterprise but also to help retain and strengthen U.S. leadership in two DOE mission areas: HPC-enabled M\&S and nuclear energy. CASL is currently completing its last year of a five-year Phase 1 period and has been granted a second five-year Phase 2 (2015-2019) of execution. Founding partner institutions in CASL include four DOE national laboratories (Idaho, Los Alamos, Oak Ridge [lead partner] and Sandia), three universities (Massachusetts Institute of Technology, Michigan and North Carolina State), and three organizations from the industrial sector (Electric Power Research Institute, Tennessee Valley Association, and Westinghouse Electric Company). In addition, over a dozen additional institutions provide key contributions to CASL via single Principal Investigator participation. More information on CASL can be found at www.casl.gov, including access to the many hundreds of technical reports CASL staff have generated since 2010.

\footnotetext{
${ }^{1}$ Consortium for the Advanced Simulation of Light Water Reactors (CASL)

Modeling and Simulation (M\&S)

Chalk River Unidentified Deposits (CRUD)

CRUD Induced Localized Corrosion (CILC)

CRUD Induced Power Shift (CIPS)

Pellet-Clad Interactions (PCI)

Grid-to-Rod Fretting (GTRF)

Reactivity-Initiated Accident (RIA)

Loss of Coolant Accident (LOCA)
} 
M\&S technology has long been a mainstay in the nuclear industry; it informs consequential nuclear power operational and safety decisions. The slow evolution of commercial nuclear technology and its strong dependence on M\&S are driven by the industry's limited ability to perform frequent full-scale irradiated experiments due to cost, safety and feasibility, and economic uncertainties. Even industry's advanced light water reactors (LWRs) rely on the current nuclear industry M\&S technology, which, though continuously improved and central to the industry's evolution, has not sufficiently capitalized on the benefits that more precise predictive simulation and fundamental understanding offer.

Opportunities for reduced uncertainties in predicting operational and safety margins, which translate to operating cost reductions and plant lifetime extension, are lost due to the lack of technology progression and leadership in the industry's M\&S. CASL's mission is to recapture the benefits of leadership in M\&S for nuclear technology by providing coupled, high fidelity, usable capabilities needed to address LWR operational and safety performance-defining phenomena.

At the highest level, CASL's goals are to provide M\&S capabilities that will aid in improving nuclear energy's economic competitiveness and to reduce the volume of spent nuclear fuel per unit energy, achieving this while assuring nuclear safety.

2. Challenge Problems and the CASL Core Simulator

To focus CASL's development of M\&S technology, a challenge problem approach has been taken. Long standing issues, amendable in part to being addressed via application of M\&S, have been identified. Once identified, the physical phenomena associated with each challenge problem are in turn elucidated. At this point the M\&S technology of the individual physics necessary is reviewed to assure that capabilities being developed can address a wider class of problems than the selected challenge problems. An integrated R\&D program is therefore established, where individual physics M\&S technology is first developed and subsequently integrated to provide the multiphysics M\&S capabilities required to address the challenge problems.

Figure 1 depicts the challenge problems, to be discussed below, that were selected to be pursued by CASL during its Phase 1. 


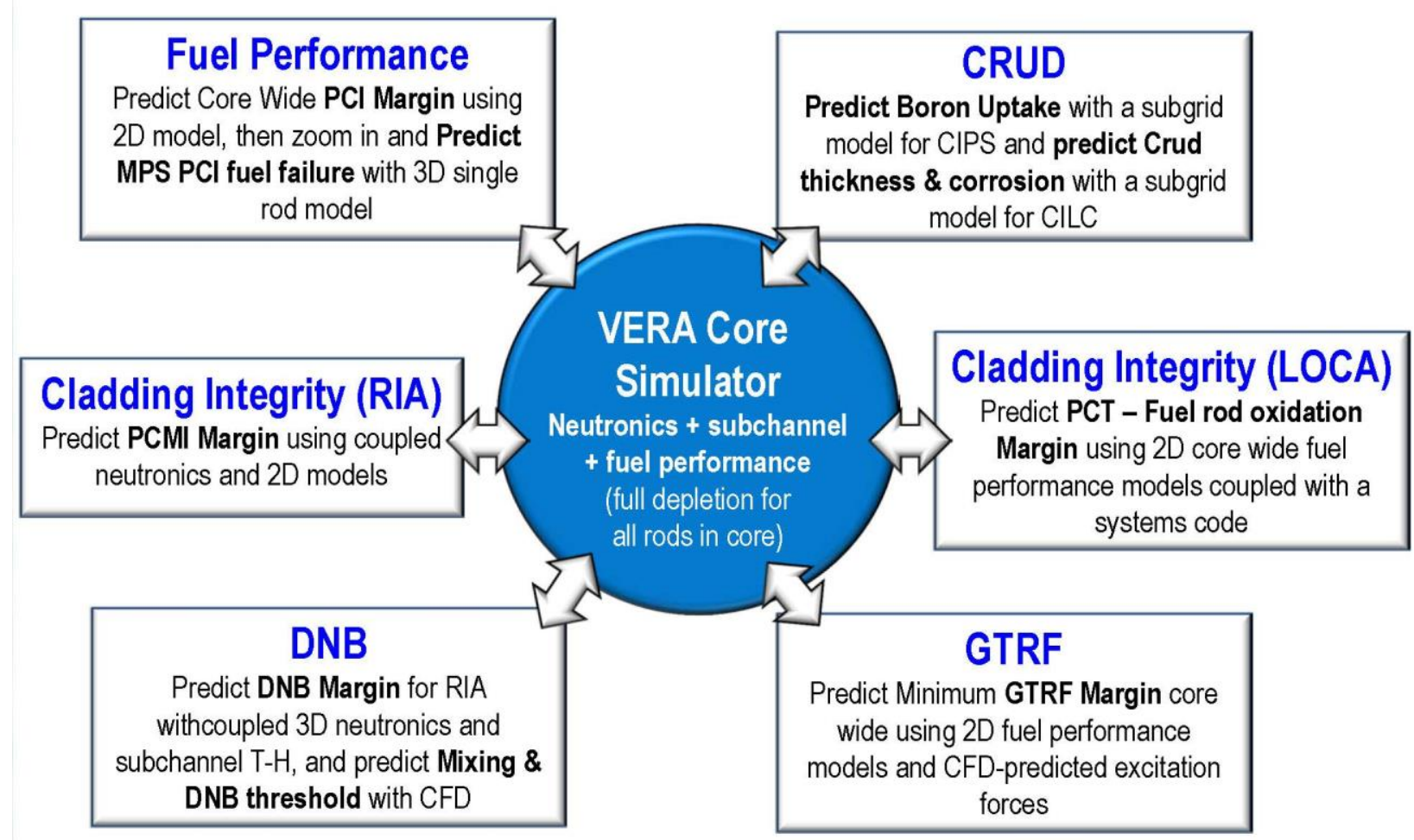

Figure 1. Challenge problems that are guiding the development of CASL's M\&S technology.

All challenge problems in Phase 1 focused on the currently operating generation of power reactors (http://www.nrc.gov/reactors/power.html), and in particular those reactors that are Pressurized Water Reactors (PWRs). The main focus is on in-core phenomena, with a sharp focus on nuclear fuel performance given its economic and safety importance. The areas of physics being addressed are radiation transport, isotopic depletion, thermal-hydraulics, fuel performance [thermo-mechanics plus materials behavior], and chemistry. Brief descriptions of each challenge problem and the core simulator are now given.

\subsection{Core Simulator}

The core simulator's prinicipal purpose is to provide the temporal and spatial nuclear interaction rates, power density, isotopic number densities, fuel and clad temperatures, and coolant temperatures and phases throughout the reactor core as a function of core exposure (e.g. fuel burnup) and operating state. The reactor core attributes are used either directly or indirectly, via linkage to additional M\&S capabilities, to address a specific challenge problem. Coupling of all the physics components noted above is required, specifically MPACT Method of Characteristics based code [2] for radiation transport, ORIGEN code [3] for isotopic depletion, and COBRA-TF subchannel based code [4] for thermalhydraulics. Recognizing that full-core calculations are required (many times quarter-core models can be used taking advantage of symmetry), M\&S fidelity compromises are required to obtain manageable computer resource requirements. For PWR core simulators, the currently utilized industry's M\&S technology is contrasted to CASL's current M\&S technology in Table I. Detailed descriptions of the physics models indicated in Table I are presented in companion articles in this special issue. To be noted 
are the computer resource requirements. An industry-class core simulator will execute on a modest engineering workstation (single to few cores and memory of 5-10 GB) with execution times of a few minutes for most applications. By contrast, CASL's core simulator, the Virtual Environment for Reactor Applications-Core Simulator (VERA-CS) [5], typically requires a cluster computer (thousands of cores and memory of 5-10 TB) with execution times of a few hours for most applications. The implication is that CASL's objective is not to replace the industry-class core simulator, but when higher fidelity is required, to provide such capability via VERA-CS. Notable is the level of spatial detail that VERA-CS captures, where nearly all components are explicitly represented geometrically - spanning axially from the bottom to the top fuel assembly nozzles and radially from outside the core's former baffle plates inward. Figure 2 illustrates the complexity of the Westinghouse Electric Company's AP1000 TM PWR Cycle 1 reactor core design, which VERA-CS is capable of representing in detail.

\begin{tabular}{|c|l|l|}
\hline Physics Model & \multicolumn{1}{|c|}{ Industry Practice } & \multicolumn{1}{c|}{$\begin{array}{c}\text { VERA Core } \\
\text { Simulator Components }\end{array}$} \\
$\begin{array}{c}\text { Neutron } \\
\text { Transport }\end{array}$ & $\begin{array}{l}\text { 3D diffusion (core), 2 energy groups } \\
\text { (core), 2D transport on single } \\
\text { assembly }\end{array}$ & $\begin{array}{l}\text { 3D transport, 23+ energy } \\
\text { groups }\end{array}$ \\
\hline $\begin{array}{c}\text { Power } \\
\text { Distribution }\end{array}$ & $\begin{array}{l}\text { nodal average with pin power } \\
\text { reconstruction methods }\end{array}$ & $\begin{array}{l}\text { pin-by-pin, either } \\
\text { homogenized or } \\
\text { explicitly modeled }\end{array}$ \\
\hline $\begin{array}{c}\text { Xenon / } \\
\text { Samarium }\end{array}$ & nodal average with correction & $\begin{array}{l}\text { pin-by-pin, either } \\
\text { homogenized or explicitly } \\
\text { modeled }\end{array}$ \\
\hline $\begin{array}{c}\text { Depletion } \\
\text { quadratic burnup correction, history }\end{array}$ & $\begin{array}{l}\text { pin-by-pin with actual core } \\
\text { conditions }\end{array}$ \\
\hline $\begin{array}{c}\text { Reflector } \\
\text { Models }\end{array}$ & 1D cross section models & actual 3D geometry \\
\hline $\begin{array}{c}\text { Thermal } \\
\text { Hydraulics }\end{array}$ & 1D assembly-averaged & subchannel with cross-flow \\
\hline $\begin{array}{c}\text { Fuel } \\
\text { Temperatures }\end{array}$ & nodal average & pin-by-pin 2D or 3D \\
\hline $\begin{array}{c}\text { Fuel } \\
\text { Performance }\end{array}$ & $\begin{array}{l}\text { empirically-based models for } \\
\text { key performance phenomena }\end{array}$ & $\begin{array}{l}\text { science-based models for } \\
\text { key performance } \\
\text { phenomena }\end{array}$ \\
\hline Target Platforms & workstation (single processing core) & $\begin{array}{l}\text { 1,000 - 300,000 processing } \\
\text { cores }\end{array}$ \\
\hline
\end{tabular}

Table I. Contrast of features of currently utilized industry-class and CASL's VERA-CS core simulators.

In addition to the MPACT radiation transport code utilized in VERA-CS, the Insilico code [6] employing Sn and SPn methodologies has been developed and utilized. To provide verification capability at the fullcore level, the continuous energy Monte Carlo code, SHIFT [7] has been developed for this purpose. 


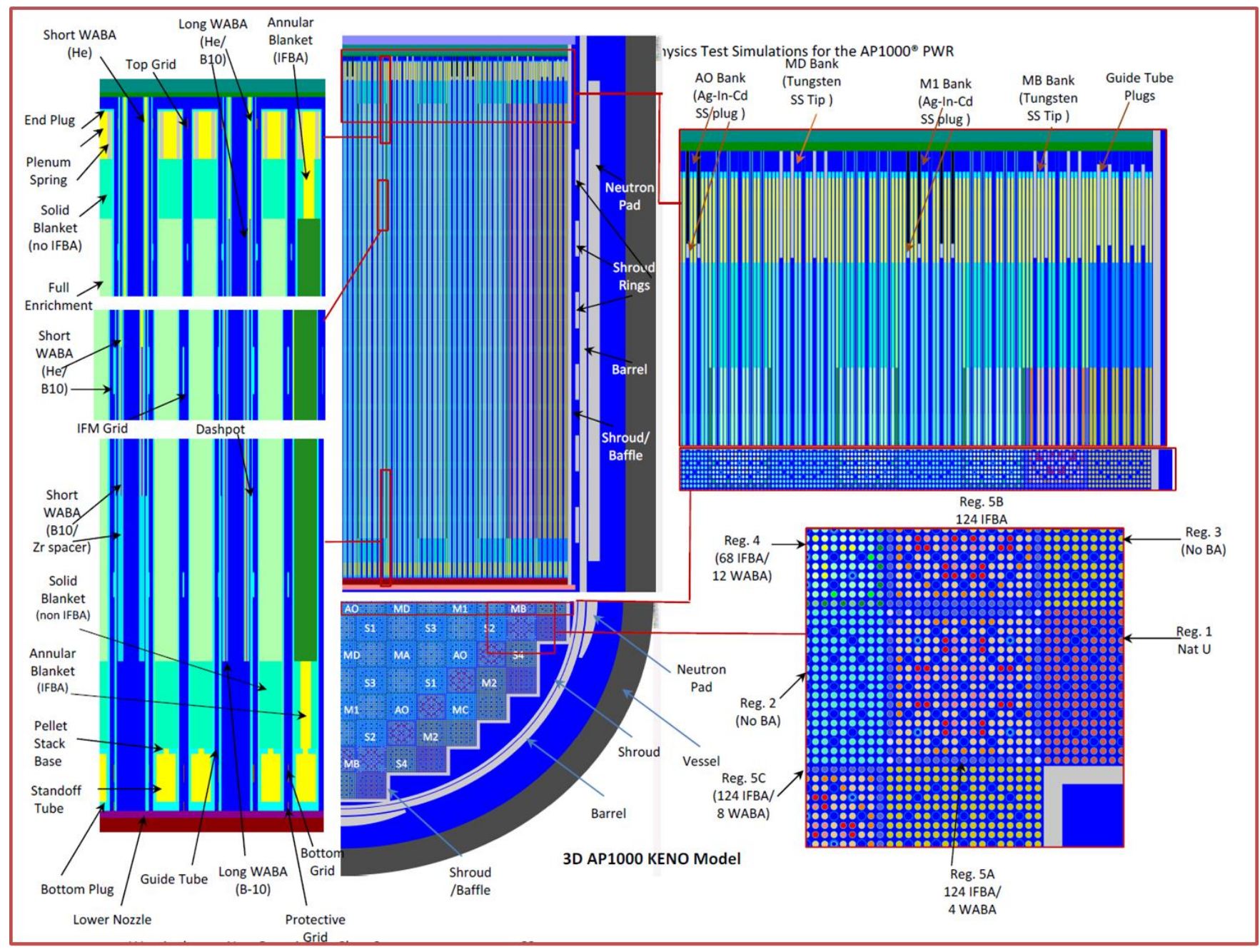

Figure 2. Illustration of the Westinghouse Electric Company's AP1000 ${ }^{\mathrm{TM}}$ PWR Cycle 1 reactor core design [courtesy of Westinghouse Electric Company, LLC].

2.2 Chalk River Unidentified Deposits (CRUD)

CRUD refers to the deposition of corrosion products, mainly from nickel and iron, on the surface of fuel rods. Figure 3 depicts CRUD deposition.

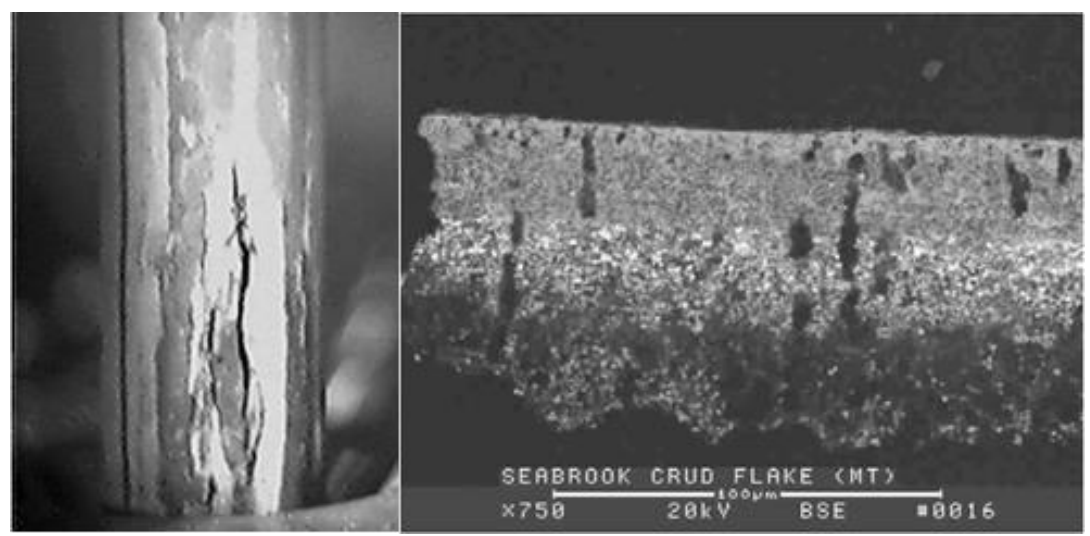


Figure 3. Picture of CRUD formation and resulting clad failure on a PWR fuel rod and CRUD scrap flake [courtesy of Westinghouse Electric Company, LLC].

In areas of the fuel clad surface where subcooled boiling occurs, particulate and soluble corrosion products in the core coolant plate out on the fuel clad surface [8]. The CRUD deposited layer causes increased fuel clad surface temperatures due to increase thermal resistance, which in turn causes accelerated corrosion of the zirconium alloy used for the fuel clad [CILC = CRUD Induced Localized Corrosion]. Also lithium borate, present due to the soluble boron in the coolant used for reactivity control (boron is a good thermal neutron capturer), accumulates in the CRUD layer, which in turn causes anomalies in the core's power distribution [CIPS = CRUD Induced Power Shift] and reactivity behavior that are adverse for certain postulated accidents. To model and predict CIPS, core-wide resolution of relevant length scales (e.g., fuel pins) is required. This implies utilization of VERA-CS with incorporation of CASL's CRUD chemistry model, MAMBA [9]. To model CRUD chemistry at the coolant/fuel cladding surface, corrosion product creation from corrosion of piping and vessels and erosion of the CRUD layer, solubility in the coolant, deposition in forming the CRUD layer, and associated overall mass balance must be addressed. This has necessitated microscale (e.g. density functional theory calculations for chemical thermodynamic and surface activation energy), mesoscale (e.g. MAMBA-BDM [10] to address two-phase flow, boiling and deposition in porous media with chimneys) and macroscale (e.g. MAMBA) M\&S capabilities, as described in accompanying articles. For a CILC analysis, a more detailed thermalhydraulic modeling capability is required than can be provided by COBRA-TF, VERA-CS's thermalhydraulic model based upon a two phase-three field, subchannel model. Utilizing Computational Fluid Dynamics (CFD) enables more accurate predictions of subcooled boiling and clad surface temperature, and via models of near wall turbulence force contribution to CRUD layer erosion. The implication is that for CILC analysis, coupling of CFD, provided by CASL's Hydra-TH CFD code [11], MAMBA chemistry code, and a fuel clad oxidation model is required. Given the higher computational resource requirement of CFD, a coupled capability (CFD+CRUD+Radiation Transport+Fuel Performance) can be employed only to analyze a limited number of rods within a fuel subassembly or assembly, with those rods identified as being susceptible to CIPS in the course of completing the core-wide CIPS analysis.

\subsection{Fuel Performance}

For this challenge problem, Pellet-Clad Interactions ( $P C I)$ is CASL's M\&S focus, while recognizing that capabilities developed for $\mathrm{PCI}$ provides a broader $\mathrm{M} \& \mathrm{~S}$ foundation for addressing fuel performance during Loss of Coolant Accidents (LOCAs) and Reactivity-Initiated Accidents (RIAs). PCI can result in vertical, through-wall cracks in the clad as depicted in Figure 4. 


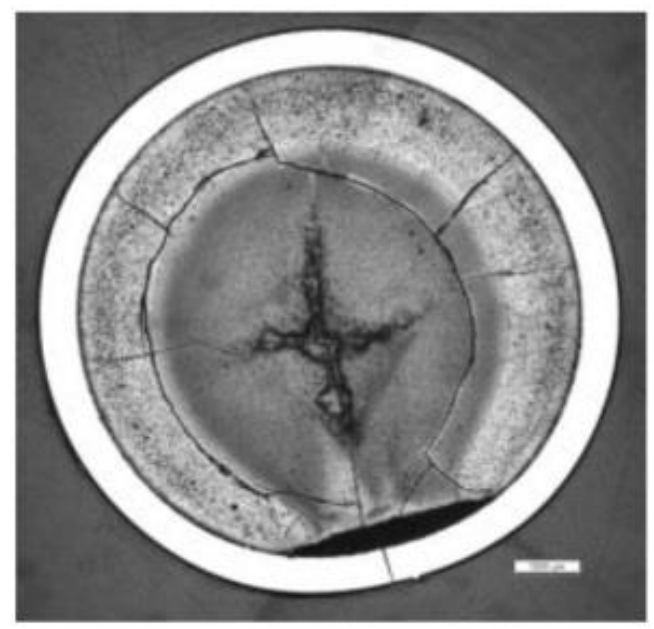

Figure 4. Picture of a PCI failure due to a missing pellet surface [12].

It occurs when the gap between the clad inner wall and fuel pellet disappears and the fuel pellet fairly rapidly expands more than the fuel clad due to heatup, resulting in high clad stresses. Two conditions lead to higher stresses. If a missing pellet surface (MPS) (e.g. chip) condition exits as a result of a manufacturing defect, high stresses occur in the clad where contact with the edges of the missing surface occur. The other stress intensifier occurs because of the cracks that form in the ceramic fuel pellet due to thermal stresses. If an axial segment portion of a fuel rod operates for an extended time period at reduced power, the associated lower fuel pellet temperature will lead to a smaller diameter pellet due to less thermal expansion. Since zircaloy clad has an enhanced creep rate in an irradiation environment and net pressure forces are directed inward, the clad will creep down to make firm contact with the fuel pellet. If now a local power increase occurs rapidly, such as would occur with repositioning a control rod, the reverse occurs except that there is inadequate time for clad creep to occur to reduce stress intensity, so vertical cracks occur. Stress corrosion cracking induced by the iodine fission product further accelerates the cracking.

M\&S capabilities required to predict the onset of $\mathrm{PCl}$ include those found in a core simulator and fuel performance code. VERA-CS provides the needed capabilities of a core simulator to be used to assess $\mathrm{PCl}$, in particular its capability to predict fuel pin-resolved power and isotopic number densities.

However the COBRA-TF thermal-hydraulic model has limited capability to model only the thermal aspect of fuel performance, with material and structural aspects of fuel performance addressed by a limited set of property lookup tables. CASL has addressed this by replacing the fuel pin thermal model in COBRA-TF with CASL's fuel performance code, BISON-CASL [13]. BISON-CASL is a derivative of the Idaho National Laboratory (INL) BISON code [14], which has been developed using the INL MOOSE framework (mooseframework.org), which is an environment for building finite element solvers with multiphysics solution capability. BISON-CASL augments BISON via utilization of industry-developed relevant fuel material behavior models. The BISON-CASL code employs a number of material behavior models for the uranium dioxide fuel pellet and zircaloy clad to obtain the material properties required when solving the coupled thermo-mechanics equations. Several of these material models, which are at the macroscale when utilized by BISON-CASL, have been developed utilizing microscale models (e.g. molecular 
dynamics) and mesoscale models (e.g. viscoplastic self-consistent model [15]). For core-wide analysis using VERA-CS coupled to BISON-CASL, referred to as TIAMAT [16], a two-dimensional $(r, z)$ fuel performance model is employed to reduce computational burden. Having identified the $\mathrm{PCl}$ susceptible fuel rods from the core-wide analysis, axial segments of the susceptible fuel rods are subsequently analyzed utilizing a three-dimensional $(r, z, \Theta)$ BISON-CASL model, differing from current industry practice [17] in both incorporating a fuel performance model in the core-wide analysis and utilizing a threedimensional fuel performance model in the detailed analysis.

\subsection{GTRF}

Grid-to-Rod Fretting denotes excessive wear of the fuel clad due to contact, both impact and sliding, between the fuel clad and the spacer grids which support the fuel rods [18]. Figure 5 depicts one of about a half dozen spacer grids holding together the fuel rods of a fuel assembly, of which there is anywhere from 120 to 250 fuel assemblies in a power reactor core depending upon the thermal power rating.
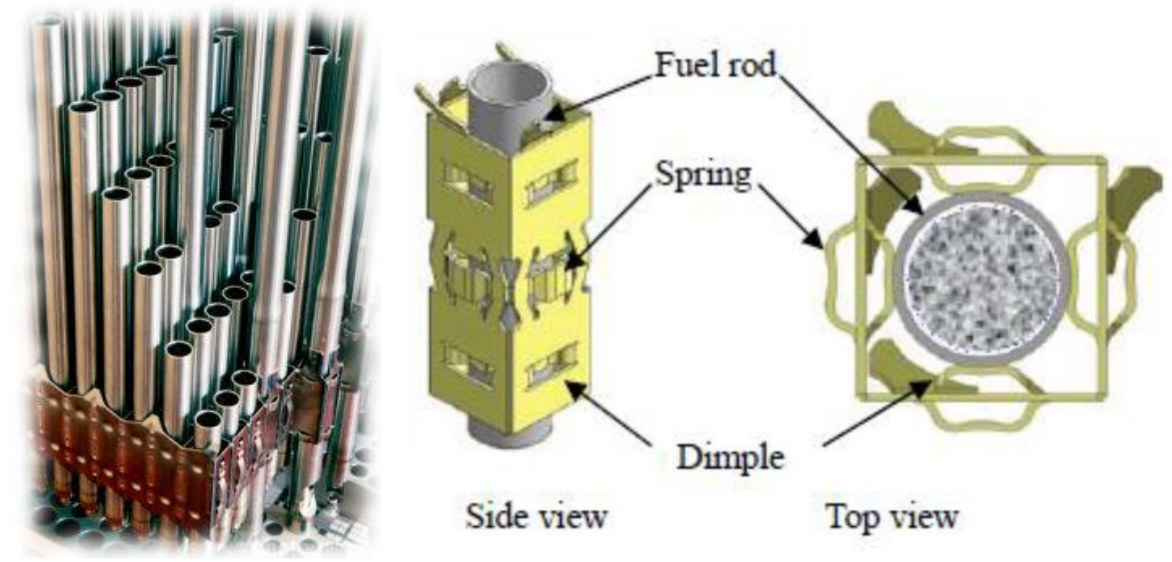

Side view

Top view

Figure 5. Illustration of a spacer grid holding fuel rods for a PWR fuel assembly and a blowup of a single cell [courtesy of Westinghouse Electric Company, LLC].

A GTRF induced through clad wall failure is shown in Figure 6. 


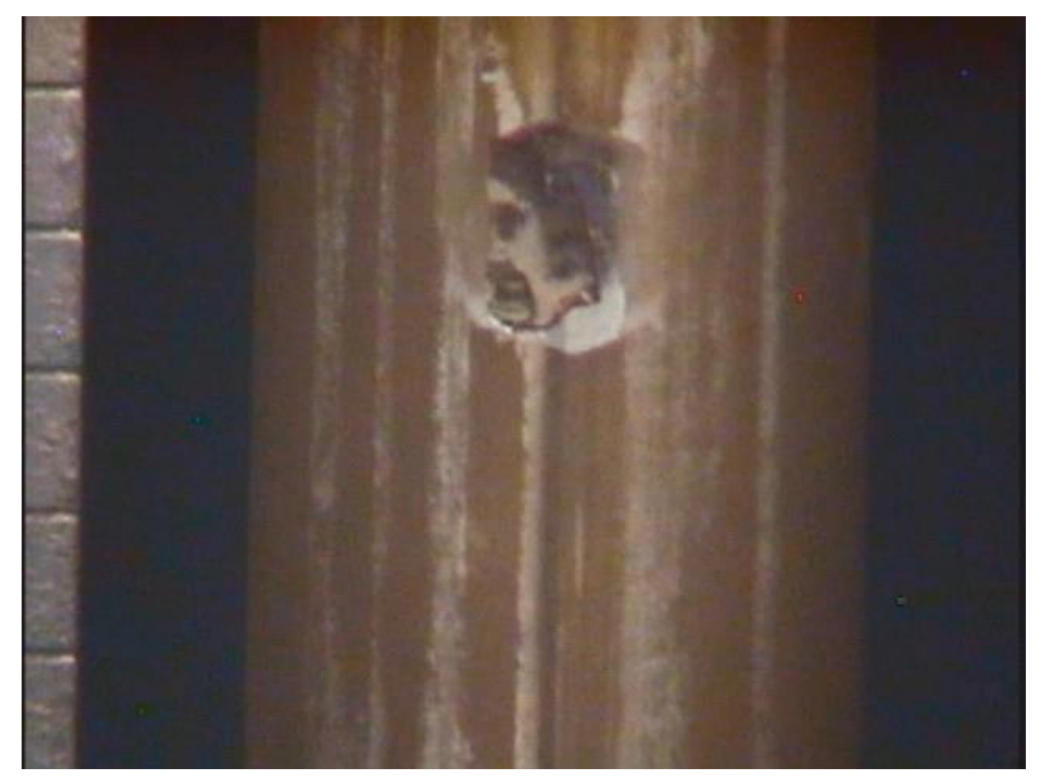

Figure 6. Picture of clad failure due to GTRF for a PWR fuel rod [courtesy of Westinghouse Electric Company, LLC].

Two conditions are required to experience excessive clad wear. The first condition is a gap opening between the fuel clad and the spacer grid. This occurs due to both growth and creep of the spacer grid and creep down of the fuel clad at some point in a fuel assembly's lifetime. The second condition is the fuel rod vibration amplitude being sufficient to cause clad-spacer grid contact. The vibration, usually in the range of $30-50 \mathrm{~Hz}$, is induced by lateral turbulent fluid forces promoted by the mixing vanes on the spacer grid [19]. These mixing vanes have been engineered to sweep off subcooled boiling created steam bubbles on the fuel clad surface which, in addition to promoting heat transfer from the rod into the coolant, helps to both gain margin from a Departure from Nucleate Boiling (DNB) condition and suppress CRUD formation. Many supporting M\&S capabilities are required for addressing GTRF: core simulator; fuel performance and spacer grid response; thermal-hydraulics; structural mechanics; and clad wear and fretting. CASL's focus is on developing all these capabilities with the exception of structural mechanics, where commercial and national laboratory capability is judged capable of addressing the needs. In contrast to other challenge problems, an integrated solution capability is not being pursued; however, VERA-CS core simulator, MOOSE-BISON fuel performance, Hydra-TH thermalhydraulics, and CASL-developed wear and fretting models will provide the basis for interoperability with CASL independent software providers' structural mechanics capability.

\subsection{Departure from Nuclear Boiling (DNB)}

DNB is the limiting critical heat flux phenomena for the majority of accidents that are postulated to occur in PWRs. CASL's focus on a DNB M\&S capability is two pronged. One prong is to continue to utilize the industry's current practice of subchannel thermal-hydraulics combined with experimentally developed DNB correlations. CASL's approach relative to current industry practice is the fidelity of the individual physics components making up the core simulator, VERA-CS, as indicated in Table I, and the employment of nonlinearly consistent multiphysics coupling. The second prong being pursued, 
considerably more challenging, is the development of a capability to predict the onset of DNB utilizing Multiphase-CFD (M-CFD). This capability, yet to be established, builds upon the subcooled boiling/bubble flow capabilities that are being developed in Hydra-TH in support of the CRUD challenge problem. To date improvements have been made in several closure models (i.e. subcooled boiling energy partitioning and lift force) based upon experiments and Direct Numerical Simulations (DNS) (see Figure 7).

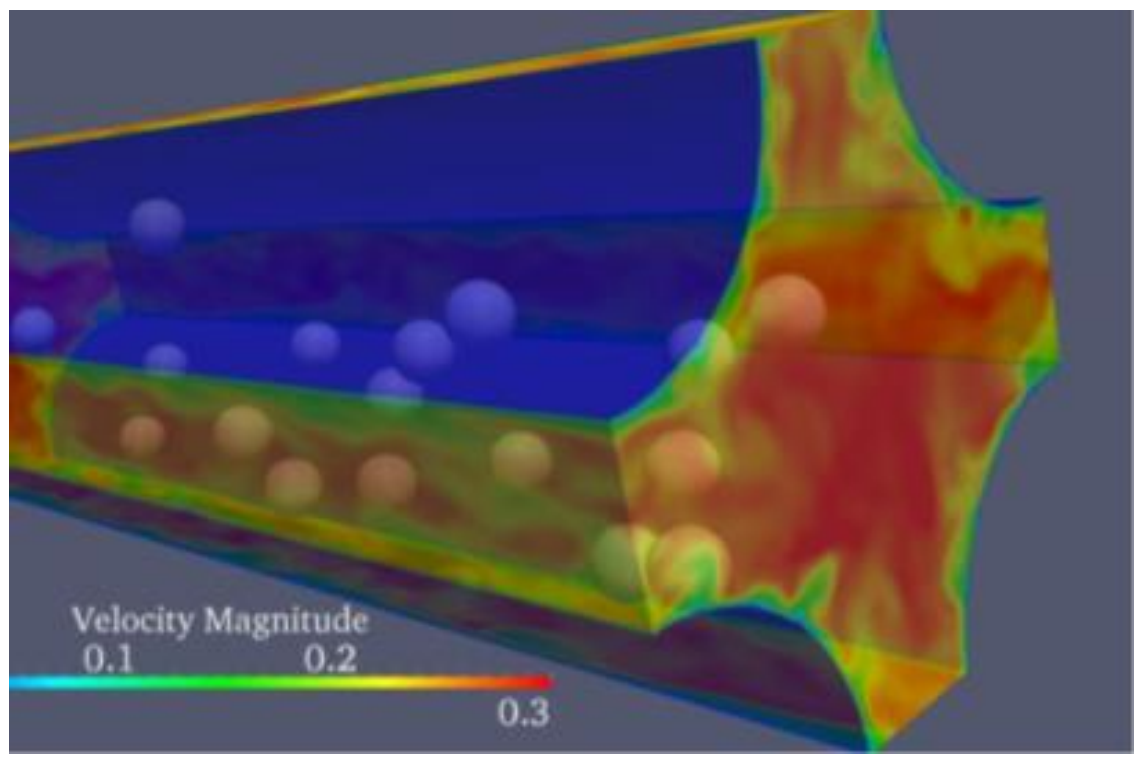

Figure 7. Illustration of vapor bubbles developed in the flow channel between fuel rods as conditions at the rod surface evolve [20].

\subsection{Reactivity-Initiated Accident (RIA)}

For a PWR, the RIA of interest is the ejected control rod accident initiated from various power levels. The power level at initiation of the RIA impacts the reactivity worth of the ejected rod and the time from accident initiation to when negative reactivity feedback from sensible heat affects the prompt critical transient [21]. Fuel clad failure is postulated to occur by several mechanisms, with the two prominent mechanisms being DNB and clad rupture due to high strain from fuel pellet expansion. Figure 8 depicts clad fracture induced by RIA test conditions. 


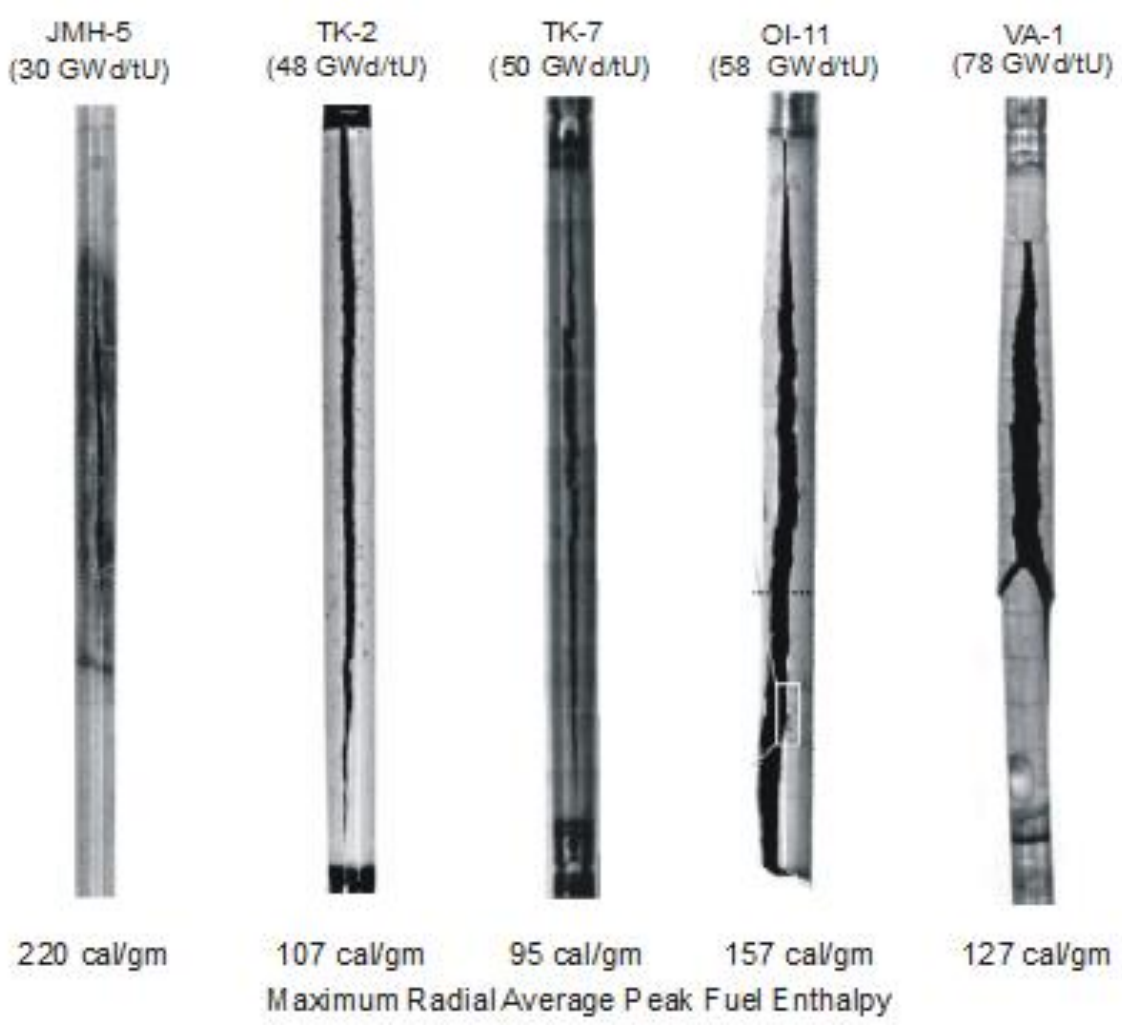

Figure 8. Clad fracture during RIA testing [22].

Upon clad rupture, hot fuel particles can be ejected into the coolant, causing a so-called "steam explosion" due to the high surface area to volume ratio of the ejected particles. Concerns about coolability also arise if catastrophic clad failure occurs. Key conditions influencing clad rupture include levels of oxidation (both at RIA initiation and during the transient), hydrogen pickup, and grain orientation. Likewise, ejected fuel particle size and quantity is influenced by the rim effect, which denotes the preferential buildup of plutonium oxide near the pellet's surface due to spatial selfshielding. This buildup changes the microstructure and results in greater accumulation of gaseous fission products in the rim area, both contributing to increased quantity of smaller particle sizes being ejected. Four key M\&S capabilities are therefore important for RIA:

- Fuel rod attributes (e.g. clad corrosion and hydrogen pickup, and rim structure) at the start of the transient;

- Fuel rod performance regarding rupture and particle ejection;

- Onset of DNB under severe transient conditions; and

- Transient simulation coupling core simulator, fuel performance, and system transient response. CASL has chosen not to attempt to model fuel performance after the onset of failure, e.g. particle ejection and subsequent steam explosion. VERA-CS and BISON-CASL have both made progress in developing capabilities to simulate a RIA, however much work remains to be completed. Of particular note is the viscoplastic self-consist model of clad growth and creep, which is reported upon in this issue.

2.7 Loss of Coolant Accident (LOCA) 
LOCA's have played a significant role in nuclear safety, and in many cases impose the limit of the core's power rating. To improve the M\&S technologies used in analyzing the LOCA challenge problem, advances in thermal-hydraulics and fuel performance are required. CASL's principal LOCA focus is on fuel performance, hence utilizing existing thermal-hydraulic capabilities to obtain time-dependent coolant boundary conditions required for the fuel performance simulation. Further, CASL's fuel performance focus is being restricted to LOCA blowdown phase (i.e. time over which the primary coolant circuit depressurizes) and uncovery phase (i.e. time over which coolant liquid level is below the core), and does not address the reflood phase (i.e. time over which coolant liquid level is rising up in the core) when fuel quenching occurs. Common to the RIA challenge problem, mechanisms that govern clad ballooning and oxidation need to be addressed via M\&S capabilities. Ballooning during large break LOCAs is promoted by the rapid depressurization of the primary coolant circuit, increasing the pressure difference across the clad. Figure 9 shows clad rupture that occurred under LOCA representative test conditions.

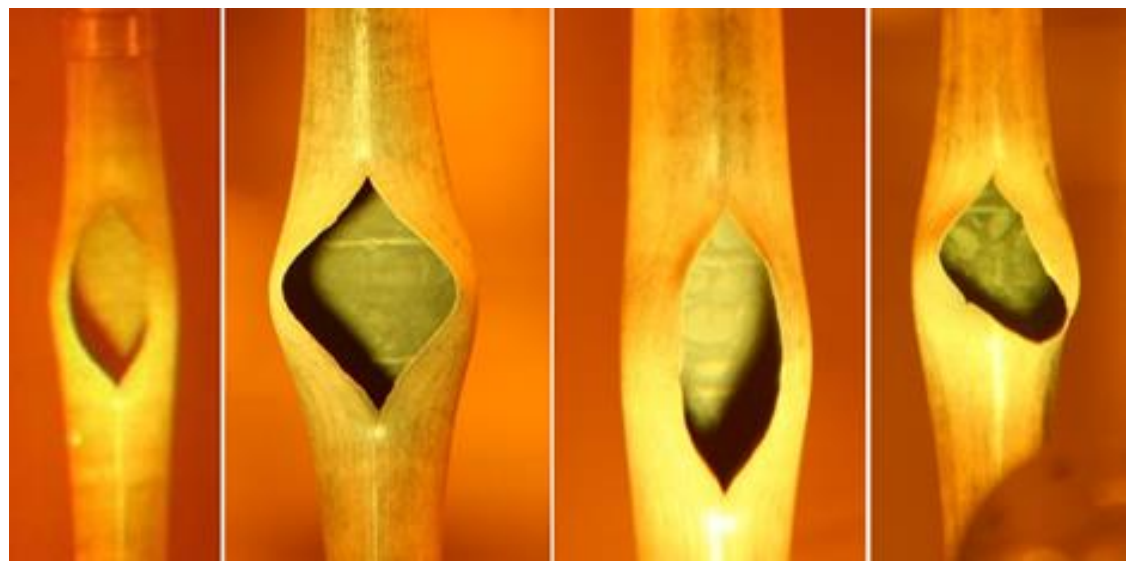

Figure 9. Clad fracture that results from testing under LOCA conditions [23].

The rate of oxidation is not only influenced by the clad temperature, but also by the amount of hydrogen pickup (i.e. retention of hydrogen in clad originating from clad corrosion), with increasing pickup resulting in enhanced permeability and penetration of oxygen into the clad (hence increased oxidation). Breakaway oxidation, the result of cracks forming at elevated temperatures in the oxide layer of the clad, thereby providing paths for oxygen intake, must also be addressed in fuel performance M\&S. To date CASL has focused on advancing material models, with the exception of the viscoplastic self-consistent model, so much work remains.

An additional requirement on CASL physics models is the ability to complete uncertainty quantification UQ) of Quantities of Interest (Qol), which in turn implies the need for the ability to complete data assimilation (i.e. calibration) [24], verification [25] and validation (V\&V). Rather than determining that the value of a Qol is not exceeded with a $95 \%$ probability at a $95 \%$ confidence interval, as used in nuclear safety analysis, CASL's objective is to predict the probability density function of the Qol. Given the large computer execution times associated with VERA-CS and other CASL M\&S technology, large number of parameters, multiphysics nature and nonlinear behaviors, the main focus of CASL's work has been on dimensional reduction, generation of surrogate models, and acceleration of sampling based uncertainty quantification and data assimilation methods. The resulting software developments have 
been incorporated when possible into the Dakota code's capability [26]. Development of adjoint methods to obtain sensitivity coefficients has also been completed, as exemplified by the Drekar CFD code [27].

\section{Summary and this Issue's Organization}

The application of flexibility and agility as a key Hub concept has spurred technical accomplishments, insights and innovations beyond those established within CASL's work plan, accelerating, focusing, and deepening the research. For example, the development of VERA's core simulator as a dedicated module was not initially envisioned; however, it was soon recognized that this combination of components provides a central capability for reactor simulation, and this innovation now offers a powerful toolset for high fidelity LWR simulation for VERA users and is in itself a technology step change, as illustrated in Table I. As another example, the work on CRUD evolution and growth models uncovered the potential existence of a different precipitant that had previously not been considered by the industry models; this compound was subsequently observed in the field. Also, the effort to couple CASL's heterogeneous physics codes inspired the development of new approaches to data transfer and numerical solution schemes to provide computationally efficient results that are now being adopted elsewhere. As a final example, CASL's experimental, theoretical, and DNS studies of bubbly flow and boiling heat transfer led to renewed vision, understanding, and ultimately a new formulation for sub-grid closures to address this high-uncertainty phenomenon in multiphase flow models.

The brief descriptions presented in this article of the challenge problems and core simulator is intended to provide a basis to conceptually integrate the articles in this issue. The immediately following article focuses on CASL's Virtual Environment for Reactor Applications (VERA). This article is then followed by articles on single physics: six articles on radiation transport codes, one article on CRUD formation, one article on fuel performance aspect of clad growth and creep, and three articles on CFD. The next two articles address aspects related to multiphysics integration. This issue is concluded with three articles covering the topics of adjoint CFD, data assimilation and verification.

Acknowledgement: Members of the CASL team, whom the authors are most appreciative of, performed the work reported upon in this manuscript. The authors acknowledge the strong support and management provided by the Office of Nuclear Energy of the U.S. Department of Energy. This research was partially supported by the Consortium for Advanced Simulation of Light Water Reactors, an Energy Innovation Hub for Modelling and Simulation of Nuclear Reactors under U.S. Department of Energy Contract No. DE-AC05-00OR22725.

\section{References}

[1] What Are the Energy Innovation Hubs?, Energy.gov site, (2010). http://energy.gov/articles/whatare-energy-innovation-hubs

[2] Benjamin Collins, Shane Stimpson, Blake W. Kelley, Mitchell T.H. Young, Brendan Kochunas, Edward W. Larsen, Thomas Downar, Andrew Godfrey, Three-dimensional nuclear reactor core simulations of the boltzmann transport equation with the 2D/1D method using MPACT, J Comput Phys (this issue). 
[3] A. G. Croff, "ORIGEN2: A versatile computer code for calculating the nuclide compositions and characteristics of nuclear materials," Nucl. Technol. 62 (1983) 335.

[4] Robert K. Salko, Maria N. Avramova, CTF Theory Manual, Pennsylvania State University, November, 2014.

[5] J. Turner, R. Summers, M. Sieger, The Virtual Environment for Reactor Applications (VERA): Design and Architecture, J Comput Phys (this issue).

[6] Steven P. Hamilton, Thomas M. Evans, Gregory G. Davidson, Seth R. Johnson, Tara M. Pandya, Andrew T. Godfrey, Hot zero power reactor calculations using the Insilico code, J Comput Phys (this issue).

[7] Tara M. Pandya, Seth R. Johnson, Thomas M. Evans, Gregory G. Davidson, Steven P. Hamilton, Andrew T. Godfrey, Implementation, capabilities, and benchmarking of Shift, a massively parallel Monte Carlo radiation transport code, J Comput Phys (this issue).

[8] J. Deshon, D. Hussey, B. Kendrick, J. McGurk, J. Secker, M. Short, Pressurized Water Reactor Fuel Crud and Corrosion Modeling, JOM Journal of the Minerals Metals and Materials Society, 63(8), (2011) 64-72.

[9] B. Kendrick, C. Stanek, M. Short, MAMBA (MPO Advanced Model for Boron Analysis), Development for CASL: Update and Applications. Presented at the EPRI-PTAC Meeting, San Antonio, TX (2014) Retrieved from http://www.casl.gov/docs/MAMBA ptacFeb2014.pdf

[10]Miaomiao Jin, Michael Short, Multiphysics modeling of two-phase film boiling within porous corrosion deposits (CRUD), J Comput Phys (this issue).

[11]Mark A. Christon, Jozsef Bakosi, Balu Nadiga, Markus Berndt, Marianne M. Francois, Alan K. Stagg, Yidong Xia, Hong Luo, A hybrid incremental projection method for thermal-hydraulics application, J Comput Phys (this issue).

[12]Y. Aleshin, C. Beard, G. Mangham D. Mitchell, E. Malek, M. Young, The Effect of Pellet and Local Power Variations on PCI Margin, Proceedings of Top Fuel 2010, Orlando, , Paper 41, 2010.

[13]R. Montgomery, D. Sunderland, C. Stanek, B. Wirth, N. Capps, R. Williamson, Peregrine: Advanced modeling of pellet-cladding interaction ( $\mathrm{PCl}$ ) failure in LWRs," Proceedings of the TopFuel 2012 Reactor Fuel Performance Meeting, Manchester, U.K., 2012.

[14]J. D. Hales, R. L. Williamson, S. R. Novascone, G. Pastore, B. W. Spencer, D. S. Stafford, K. A. Gamble, D. M. Perez, W. Liu, BISON Theory Manual - The Equations Behind Nuclear Fuel Analysis, BISON Release 1.1, INL/EXT-13-29930 Rev. 1, October 2014.

[15] Robert Montgomery, Carlos Tomé, Wenfeng Liu, Alankar, Gopinath Subramanian, and Chris Stanek, Use of multiscale zirconium alloy deformation models in nuclear fuel behavior analysis, J Comput Phys (this issue).

[16]Kevin Clarno, Roger Pawlowski, Incorporate MPACT into TIAMAT and demonstrate pellet-clad interaction (PCI) calculations, CASL Milestone Report: L3:PHI.CMD.P10.01(2014).

[17]J. Rashid, S. Yagnik, R. Montgomery, Light Water Reactor Fuel Performance Modeling and MultiDimensional Simulation, JOM Journal of the Minerals Metals and Materials Society, 63(8) (2011) 8188.

[18]R. Lu, Z. Karoutas, T. Sham, CASL Virtual Reactor Predictive Simulation: Grid-to-Rod Fretting Wear, JOM Journal of the Minerals Metals and Materials Society, 63(8) (2011) 53-58. 
[19]Mark A. Christon, Roger Lu, Jozsef Bakosi, Balu Nadig, Zeses Karoutas, Markus Berndt, Large-eddy simulation, fuel rod vibration and grid-to-rod fretting, J Comput Phys (this issue).

[20]Jun Fang, V. Mishra, Igor A. Bolotnov. Interface tracking simulation of two-phase bubbly flow in a PWR subchannel, International Embedded Topical Meeting on Advances in Thermal Hydraulics (ATH '14), Reno, NV, 2014.

[21]K. O. Ott, R. J. Neuhold, Introduction to Nuclear Reactor Dynamics, American Nuclear Society, La Grange Park, IL, 1985.

[22]R. Montgomery, "Industry Information to Support Interim RIA Criteria," Public Workshop on Interim RIA Criteria, US NRC Report ML063190105, November 9, 2006.

[23]R. Raynauad, "Fuel Fragmentation, Relocation, and Dispersal During the Loss-of-Coolant A," US NRC Report NUREG-2121, March 2012.

[24]Allison Lewis, Ralph Smith, Brian Williams, Victor Figueroa, An information theoretic approach to use highfidelity codes to calibrate low-fidelity codes, J Comput Phys (this issue).

[25]William Rider, Walt Witkowski, James R. Kamm, Tim Wildey, Robust verification analysis, J Comput Phys (this issue).

[26]B. Adams, L. Bauman, W. Bohnhoff, K. Dalbey, J. Eddy, M. Ebeida, M. Eldred, P. Hough, K. Hu, J. Jakeman, L. Swiler, D. Vigil, DAKOTA: A Multilevel Parallel Object-Oriented Framework for Design Optimization, Parameter Estimation, Uncertainty Quantification, and Sensitivity Analysis: Version 5.3.1 User's Manual, SNL SAND Report, May 2013.

[27]J.N. Shadid, T.M. Smith, E.C. Cyr, T.M. Wildey, R.P. Pawlowski, Stabilized FE simulation of prototype thermal-hydraulics problems with integrated adjoint-based capabilities, J Comput Phys (this issue). 